Original Paper http://ajol.info/index.php/ijbcs http://indexmedicus.afro.who.int

\title{
Evolution des caractéristiques physico-chimiques de la graine et de l'huile de pourghère (Jatropha curcas) en fonction du degré de maturité des fruits
}

\author{
Gbetoho Noël Romaric HOUMBA ${ }^{1,2}$, Christophe Bernard GANDONOU ${ }^{1 *}$, Ayihadji Paul \\ HOUSSOU $^{3}$, Marlène CAPO-CHICHI ${ }^{4}$, Alban HOUNGBEME ${ }^{5}$ et Fernand GBAGUIDI ${ }^{5}$ \\ ${ }^{I}$ Laboratoire de Physiologie Végétale et d'Etude des Stress Environnementaux, Faculté des Sciences et \\ Techniques, Université d'Abomey-Calavi, 01 BP 526 Tri postal, Cotonou, République du Bénin. \\ ${ }^{2}$ UFR Sciences Agronomiques, Université Africaine de Technologie et de Management (UATM Gasa \\ Formation), 04 BP 1361 Cotonou, République du Bénin. \\ ${ }^{3}$ Programme Technologie Alimentaire et Agricole (PTAA), Institut National des Recherche Agricole du Bénin \\ (INRAB), BP 128, Porto-Novo, République du Bénin. \\ ${ }^{4}$ GERES Bénin, Maison Atodji, Quartier Kpokon, 02 BP 825 Bohicon, République du Bénin. \\ ${ }^{5}$ Laboratoire de Pharmacognosie, Centre Béninois de la Recherche Scientifique et Technique (CBRST) ; \\ BP 06 Oganla, Porto-Novo, République du Bénin. \\ *Auteur correspondant ; E-mail: ganchrist@hotmail.com, ganchrisl@yahoo.fr, Tél. : (00229) 97396978
}

\section{REMERCIEMENTS}

Les auteurs remercient le Groupe Energies Renouvelables, Environnement et Solidarité (GERES ONG) pour le financement de cette étude.

\section{RESUME}

Jatropha curcas est l'une des espèces végétales identifiée dans les régions tropicales comme prometteuse pour la production d'huile végétale pure ou de biodiesel. L'influence du degré de maturité des fruits de pourghère sur la qualité de la graine et de l'huile a été évaluée afin d'élaborer les bonnes pratiques de récolte. Trois degrés de maturité ont été pris en compte: fruit vert-jaune, fruit entièrement jaune et fruit entièrement noir. Les paramètres physico-chimiques considérés sont la teneur en eau des graines, leur teneur en huile, l'acidité et la densité de l'huile. Les graines issues de fruits noirs ont présenté les caractéristiques les plus intéressantes avec une teneur en eau plus faible $(9,25 \%)$, une teneur en huile plus élevée $(42,59 \%)$ et une acidité de l'huile plus faible (3,9\%). Les graines issues des deux autres types de fruit ont présenté des teneurs en eau élevées $(>25 \%)$, des teneurs en huile plus faibles $(<15 \%)$ avec des acidités de l'huile plus élevées ( $>$ $25 \%)$ et des densités plus faibles $(<0,90)$. Les caractéristiques physico-chimiques de la graine et de l'huile se sont améliorées pendant la maturation du fruit et atteignent leurs meilleures valeurs au niveau des fruits noirs excepté la densité.

() 2016 International Formulae Group. All rights reserved.

Mots clés : pignon d'Inde, récolte, huile végétale pure, biodiesel, qualité de l'huile, Bénin. 


\title{
Evolution of physico-chemical characteristics of seed and oil of jatropha (Jatropha curcas $\mathbf{L}$.) depending on the degree of maturity of fruit
}

\begin{abstract}
Jatropha curcas L. is one of vegetable species identified in tropical countries like promising for pure vegetable oil production or biofuel. The objective of this study is to evaluate the influence of physic nut fruit (Jatropha curcas) maturity degree on seed and oil quality so to work out good practice of harvest. Three degrees of maturity were taken into account: green-yellow, entirely yellow and entirely black fruit. The physicchemical parameters considered are seeds water content, seeds oil content, oil acidity and density. According to the fruit degree of maturity, seeds resulting from black fruits showed the most interesting characteristics with the lowest moisture content $(9.25 \%)$, the highest oil content $(42.59 \%)$ and the weakest oil acidity (3.9\%). The seeds resulting from the two other types of fruits showed higher moisture contents (> 25\%), lower oil contents $(<15 \%)$ with higher oil acidities $(>25 \%)$ and weaker densities $(<0.90)$. The physicochemical characteristics of seed and oil evolve during fruit maturation and reach their best values for black fruits excepted oil density. Thus, in the absence of good method of drying, black fruit is the best stage of harvest for the production of pure vegetable oil of good quality.

(C) 2016 International Formulae Group. All rights reserved.
\end{abstract}

Keywords: Jatropha curcas, harvest, oil content, acidity, quality, Benin.

\section{INTRODUCTION}

Les biocarburants constituent une des formes d'énergies renouvelables auxquelles s'intéressent les gouvernements de plusieurs pays en développement. Ils sont produits à partir de plantes sucrières (bioéthanol) ou à partir de plantes oléagineuses (biodiesel). Actuellement, environ $84 \%$ du biediesel produit dans le monde provient du colza (Carels, 2009). Le reste provient de l'huile de tournesol (13\%); de palmier à huile (1\%); de l'huile de soja et autres (2\%) (Gui et al., 2008). Le pourghère ou pignon d'Inde (Jatropha curcas), est l'une des plantes tropicales pouvant produire du biocarburant à partir d'huile végétale issue de graines. Selon un rapport de la FAO/FIDA (2010), l'utilisation du pourghère pour la production de biodiesel pourrait constituer un avantage pour les agriculteurs modestes, tout particulièrement dans les zones semi-arides et reculées des pays en développement. Malgré les vives polémiques soulevées par cette production d'agro-carburant concernant les menaces sur la sécurité alimentaire, la superficie des plantations de poughère en Afrique était estimée en 2011 à 95300 ha (Wahl et al., 2012). Le rendement en huile de la plante est de 1892 litres/ha/an en moyenne (Chisti, 2007). Cependant, Abidin et al. (2009) rapportent qu'en plus de l'absence de concurrence avec l'alimentation, le biodiesel à base de l'huile de Jatropha curcas offre une alternative commercialement viable au diesel car il a des caractéristiques physico-chimiques et des performances comparables. Cette source d'énergie biodégradable et non toxique requiert plus d'attention ces dernières décennies (Stavarache et al., 2007 ; Sarin et al., 2007; Tiwari et al., 2007), protégeant ainsi les oléagineux alimentaires comme le soja, le tournesol et le palmier à huile (Veljkovic et al., 2006) qui ont été expérimentées pour la production de biodiesel (Akowuah et al., 2012). 
Ainsi, ces bioénergies permettront de réduire en partie les importations onéreuses des hydrocarbures polluantes. Plusieurs travaux ont été effectués sur l'évaluation écologique, ethnobotanique et agromorphologique d'accessions locales ou introduites de pourghère au Bénin (Assogbadjo et al., 2009; Ahoton et al., 2011 ; Gandonou et al., 2012a ;b) de même que leur sélection et leur amélioration génétique (Ahoton et Quenum, 2012) . Cependant, très peu de travaux ont porté sur l'organisation de la récolte des fruits de pourghère. Or le mode de récolte généralement utilisé est une récolte étalée dans le temps. Cet étalement de la récolte est dû à un étalement de la maturation des fruits. Cette maturation passe par quatre stades principaux caractérisés par une coloration différente du fruit à savoir : vert-jaune, jaune, jaune-noire et noire; la maturité physiologique étant atteinte quand les fruits sont colorés en jaune (Minengu et al., 2014). La connaissance des caractéristiques physicochimiques de la graine et de l'huile à chacun de ces stades de maturation pourrait permettre de mieux organiser la récolte à travers un mode de récolte plus groupé. Les caractéristiques physico-chimiques utilisées pour apprécier la qualité d'une huile végétale à utiliser comme biocarburant sont généralement la teneur en eau, la teneur en huile, l'acidité, la densité, la viscosité, la composition en acides gras, l'énergie massique, etc.

Ce travail a pour but de contribuer à une meilleure organisation de la récolte des fruits de pourghère pour un bon rendement en huile.

\section{MATERIEL ET METHODES Matériel végétal}

Le matériel utilisé est constitué de fruits et de graines de pourghère qui ont été récoltés dans la commune de Bohicon dans le département du Zou au centre Bénin. Concernant le degré de maturité des fruits, trois degrés de maturité ont été pris en compte : fruits vert-jaunes, fruits entièrement jaunes et fruit entièrement noirs qui ont été décapsulés pour obtenir les graines. La Figure 1 montre les différents degrés de maturité des fruits de pourghère.

\section{Récolte des fruits}

Les fruits utilisés ont été récoltés à la même date et sont de la même provenance (Bohicon). Trois répétitions ont été faites pour chaque essai (type de fruit). En effet, pour l'essai, les graines ont été prélevées directement à partir des fruits fraîchement récoltés sans séchage. Ces graines ont été emballées dans une enveloppe kaki recouverte de porte fiche en plastique et gardées au frais à $-20{ }^{\circ} \mathrm{C}$ jusqu'à l'extraction.

\section{Détermination des différents paramètres physico-chimiques des graines et de l'huile Teneur en eau des graines}

La teneur en eau est déterminée à partir de la perte de masse subie par l'échantillon après chauffage dans une étuve électrique à $103 \pm 2{ }^{\circ} \mathrm{C}$, suivant la Norme Internationale NF ou norme ISO 662 et exprimée en pourcentage de masse fraîche de la graine.

\section{Teneur en huile des graines}

L'huile a été extraite à l'hexane et au soxhlet. La teneur en huile est déterminée à partir de $20 \mathrm{~g}$ d'albumen frais et exprimée en pourcentage de masse fraîche d'albumen. 


\section{Acidité de l'huile}

L'acidité d'un corps gras est le pourcentage d'acides gras libres exprimé conventionnellement en acide oléique. L'acidité de l'huile a été déterminée suivant la norme NF $\mathrm{T}$ 60-20 et exprimée en pourcentage.

\section{Densité de l'huile}

La densité relative de l'huile est le rapport de la masse d'un certain volume de l'huile à $20^{\circ} \mathrm{C}$ et la masse d'un volume égal de l'eau distillée à $20^{\circ} \mathrm{C}$. La densité de l'huile a été déterminée suivant la norme AFNOR.

\section{Analyse statistique}

Les données ont été traitées avec le logiciel Microsoft Excel 2007. L'analyse de variance à un critère de classification a été effectuée par le logiciel SPSS 16. Les moyennes ont été comparées au seuil de signification de 0,05 en utilisant le test de Student Newman Keuls (SNK).

\section{RESULTATS}

\section{Teneur en eau des graines}

La Figure 2 présente la teneur en eau des graines selon le degré de maturité des fruits. Selon le degré de maturité du fruit, les teneurs en eau des graines varient de $27,99 \%$ pour les graines issues de fruits vert-jaunes à $9,26 \%$ pour les graines issues de fruits noirs. Les graines issues de fruits jaunes ont une teneur en eau de $25,29 \%$, légèrement inférieure à celle des graines issues de fruits vert-jaune $(27,99 \%)$. La différence observée entre les teneurs en eau des trois degrés de maturité de fruits est très hautement significative $(\mathrm{P}<0,001)$. La teneur en eau des graines diminue donc en fonction de l'évolution du degré de maturité des fruits.

\section{Teneur en huile des graines}

La Figure 3 présente la teneur en huile des graines selon le degré de maturité des fruits. La teneur en huile des graines est encore appelée rendement en huile des graines. Il apparaît que les graines issues de fruits noirs ont eu le meilleur rendement en huile (plus de 40\%) comparativement aux graines issues de fruits vert-jaunes et de fruits jaunes. Ces derniers ont présenté des rendements en huile faibles (moins de 15\%) et similaires. On note une différence très hautement significative $(\mathrm{P}<0,001)$.

\section{Acidité de l'huile}

Pour mieux comparer l'acidité des huiles, on a réalisé la Figure 4 qui montre l'acidité des huiles selon l'évolution du degré de maturité des fruits.

Pour les graines issues de fruits vertjaunes et celles issues de fruits jaunes, la teneur en acide oléique des huiles a été très élevée et a dépassé $25 \%$ contrairement aux fruits récoltés au stade noir de maturité où l'acidité de l'huile extraite des graines a été faible, en dessous de 5\%. La différence observée entre l'acidité des huiles issues des trois types de graines est très hautement significative $(\mathrm{P}<0,001)$. L'acidité de l'huile diminue donc au fur et à mesure que le fruit murît et atteint sa plus faible valeur au niveau des fruits noirs.

\section{Densité de l'huile}

La Figure 5 montre la densité des huiles selon le degré de maturité des fruits. Selon le degré de maturité du fruit, la densité de l'huile extraite des graines a varié de 0,891 pour les fruits vert-jaunes à 0,985 pour les fruits noirs. L'huile extraite des graines issues de fruits jaunes a présenté une densité de 0,893 , très légèrement supérieure à la densité obtenue pour l'huile extraite des graines issues de fruits vert-jaunes $(0,891)$. Il existe de différence entre la densité de l'huile issue des fruits noirs et celles issues des autres fruits (vert-jaunes et jaunes) $(\mathrm{P}<0,05)$. 


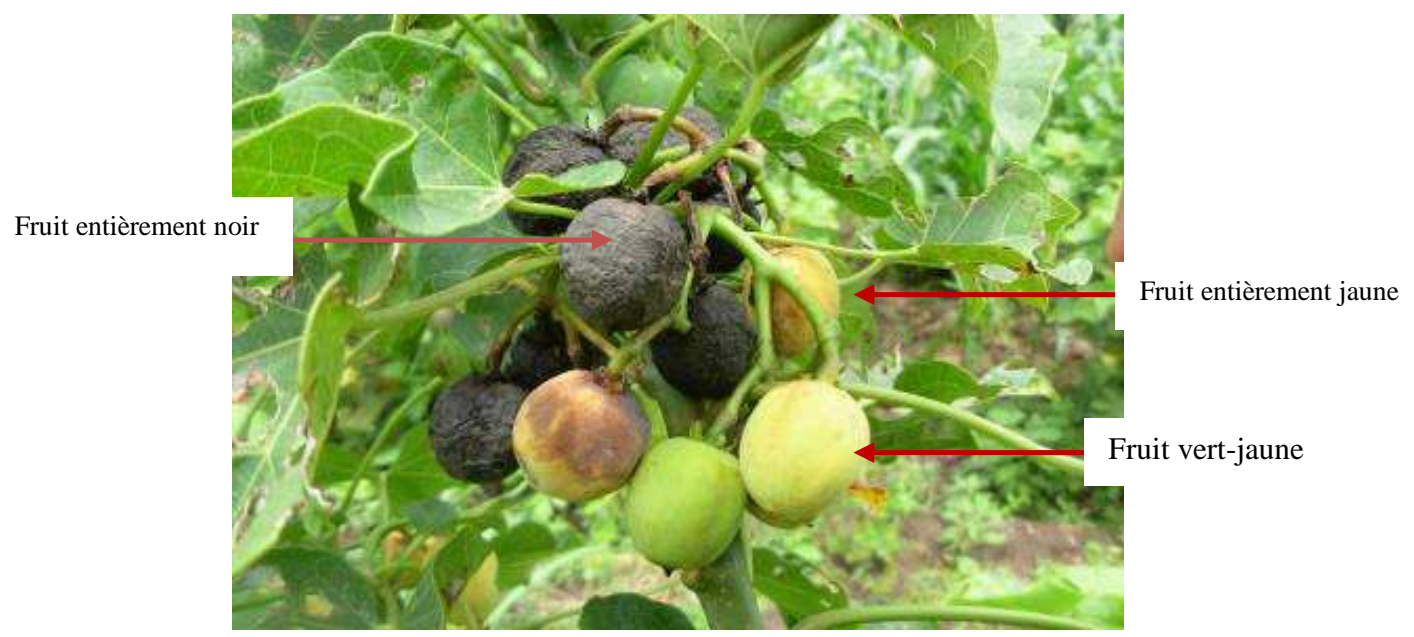

Figure 1 : Différents degrés de maturité de fruits de pourghère (Jatropha curcas).

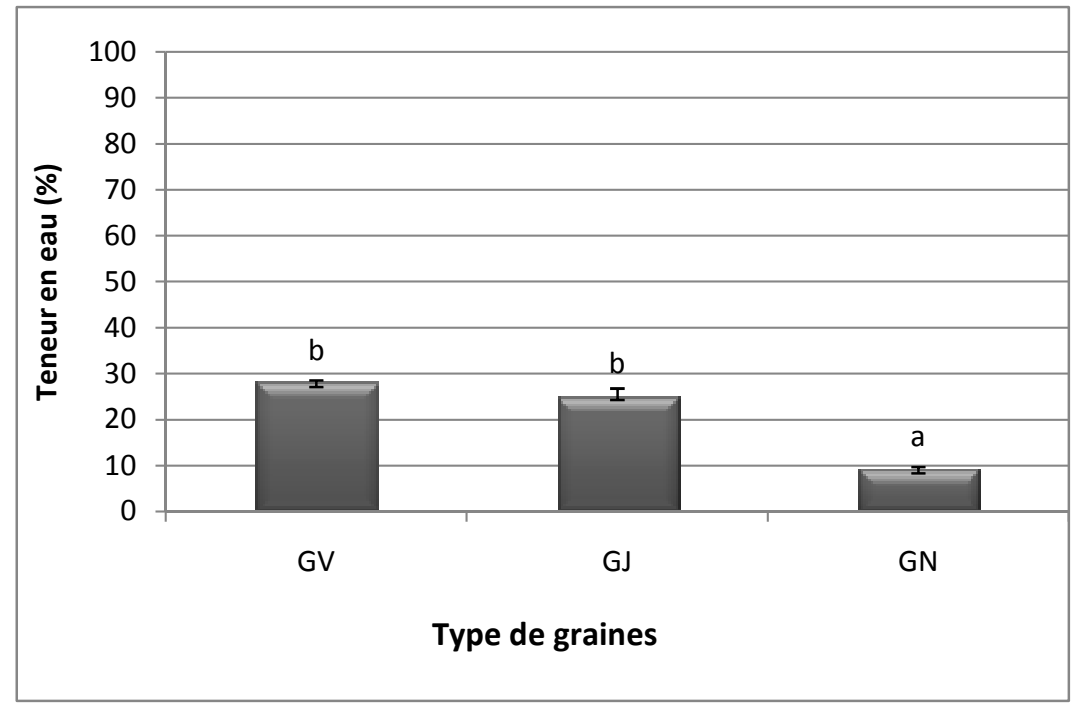

Figure 2: Teneur en eau des graines selon le degré de maturité des fruits $(n=3$; les barres verticales représentent l'erreur standard). GV : Graines issues de fruits vert-jaunes; GJ : Graines issues de fruits jaunes ; GN : Graines issues de fruits noirs 


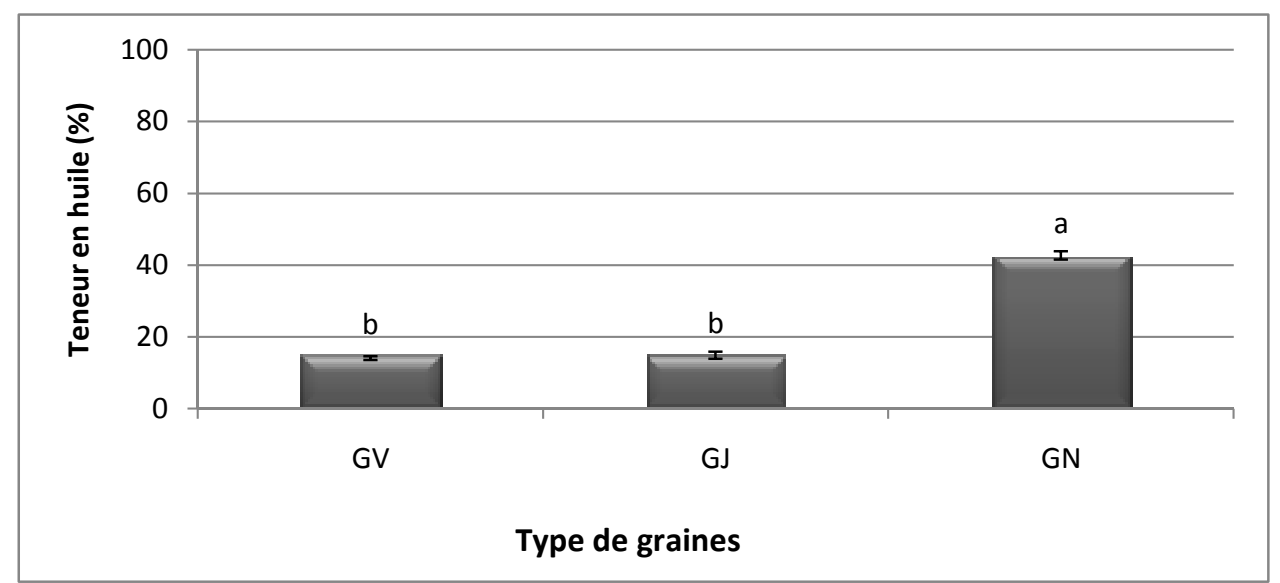

Figure 3: Teneur en huile des graines selon le degré de maturité des fruits. $n=3$; les barres verticales représentent l'erreur standard.

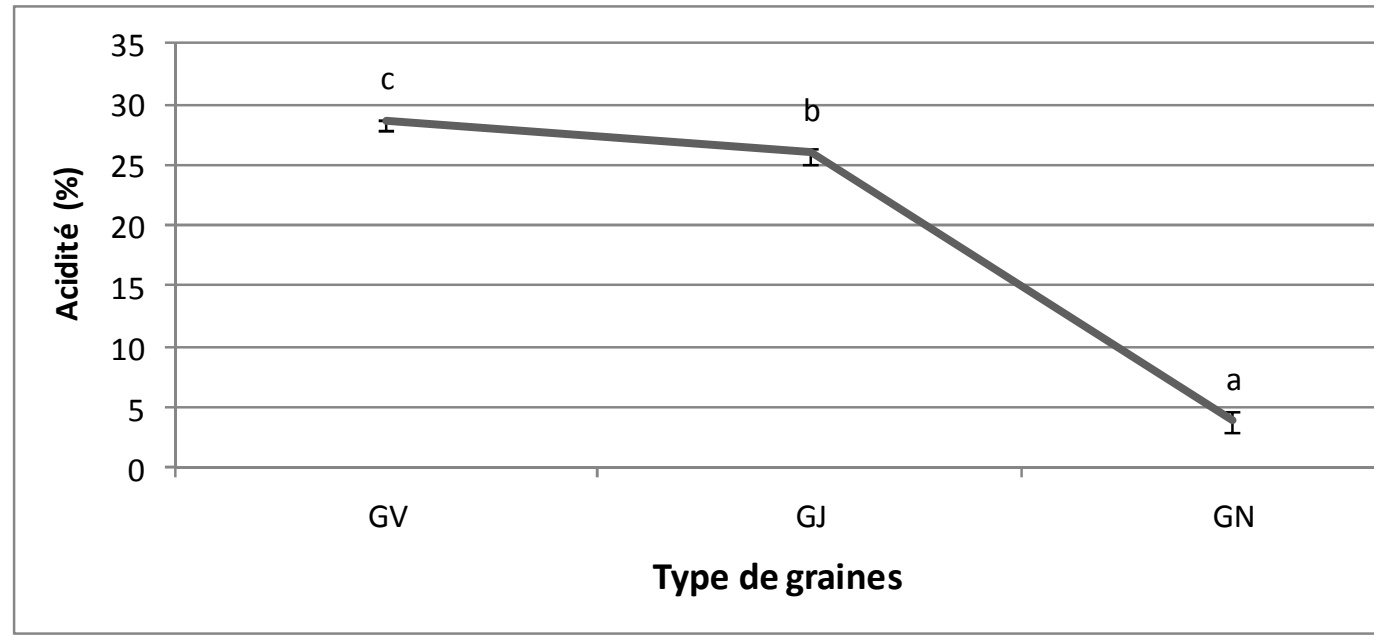

Figure 4: Acidité des huiles selon le degré de maturité des fruits. $n=3$; les barres verticales représentent l'erreur standard. 


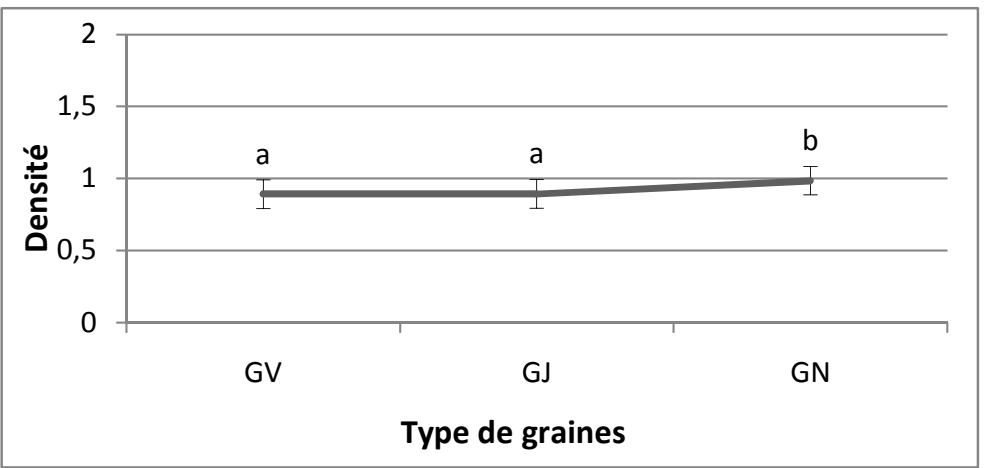

Figure 5 : Densité des huiles selon le degré de maturité des fruits. $\mathrm{n}=3$; les barres verticales représentent l'erreur standard.

\section{DISCUSSION}

La teneur en eau des graines est élevée et supérieure à $25 \%$ pour les graines issues de fruits vert-jaunes et de fruits jaunes. Elle est encore légèrement plus élevée pour les graines issues de fruits vert-jaunes et réduite à moins de $10 \%$ pour les graines issues de fruits noirs. Ces résultats montrent que la teneur en eau des graines diminue avec l'évolution de la maturité des fruits. En effet, ces fortes teneurs observées pour les deux premiers stades de maturité des fruits montrent qu'en début de maturité des fruits, les graines sont aussi très humides. Ainsi, la réduction non négligeable de la teneur en eau observée pour les graines issues de fruits noirs (plus de la moitié de la teneur en eau des autres graines) permet de dire que le stade de fruits noirs semble être le degré de stabilisation de la teneur en eau des graines. Ce stade noir de fruits est le dernier changement possible de couleur observée selon le degré de maturité des fruits. On peut donc dire que la teneur en eau des graines diminue avec l'évolution du degré de maturité des fruits. Cette évolution du degré de maturité se traduit par le changement progressif de couleur du vert au noir en passant par vert-jaune, jaune, jaune-noir. La plupart des auteurs ont rapporté des teneurs en eau comprises entre $5 \%$ et $7 \%$ mais pour des graines qui ont été séchées généralement au stade noir (Ferrao et al., 1982). Cependant, la teneur en eau obtenue $(9,26 \%)$ pour des graines issues de fruits noirs reste assez proche de 7\% (Henning, 2007).

Les teneurs en huile obtenues pour les graines issues de fruits vert-jaunes et de fruits jaunes sont faibles et inférieures à $15 \%$ alors qu'elles sont supérieures à $40 \%$ pour les graines issues de fruits noirs. On constate également que malgré les teneurs en huile voisines des graines issues de fruits vertjaunes et de fruits jaunes, la teneur en huile des graines issues des fruits jaunes est légèrement supérieure à celle des graines issues de fruits vert-jaunes. On note alors une bonne performance en huile des graines issues de fruits noirs. Ces résultats montrent que plus le degré de maturité du fruit évolue, plus on a un bon rendement en huile des graines. Ce rendement en huile obtenu pour les graines issues de fruits noirs est comparable aux rendements de la littérature. Ces rendements selon Jongschaap et al. (2007) peuvent atteindre $39 \%$ d'huile pour les graines, voire environ $55 \%$ pour les amandes selon Ferrao et 
al. (1982). Par ailleurs, les teneurs en huile obtenues peuvent s'expliquer aussi par l'influence de la teneur en eau des graines. La baisse de la teneur en huile des graines issues de fruits en début de maturité peut être liée à leur forte teneur en eau, comme cela a été rapporté par Kabutey et al. (2010). L'augmentation de la teneur en huile des graines peut donc être attribuée à la baisse de la teneur en eau des graines qui, selon Sirisomboon et Kitchaiva (2008), concerne le processus de récupération d'huile. Ceci prouve l'existence d'une relation entre la teneur en eau et la teneur en huile des graines selon le degré de maturité des fruits. De plus, avec les graines issues de fruits noirs, la teneur en huile atteint $42 \%$ pour une teneur en eau d'environ 9\%. Des observations similaires ont été faites par Sirisomboon et Kitchaiva (2008).

Concernant l'acidité des huiles obtenues à partir des graines fraîchement récoltées, on note de fortes acidités pour les graines issues de fruits vert-jaunes et de fruits jaunes (dépassent 25\%) par opposition à l'acidité largement réduite des huiles extraites des graines issues de fruits noirs (inférieure à $4 \%$ ). On note une légère supériorité d'acidité observée pour les huiles des graines issues de fruits vert-jaunes, on peut dire que l'acidité de l'huile extraite des graines de pourghère baisse au fur et à mesure que les fruits exploités passent respectivement au vertjaune, au jaune et au noir. La valeur idéale pour une huile de type carburant doit être inférieure à 1 (Diop, 2009). Ce même auteur évoque une valeur acceptable inférieure à $2 \%$ dans le biodiesel à base d'huile de jatropha. Par ailleurs, le standard allemand évoque une valeur maximale de 2 (Val Biom, 2005). On peut alors déduire que l'acidité de l'huile extraite des graines diminue avec l'évolution du degré de maturité du fruit mais reste supérieure aux valeurs acceptables.

La densité des huiles est similaire pour les fruits vert-jaunes et jaunes et restent très proches des valeurs citées dans la littérature pour les huiles végétales pures comprises entre 0,9 et 0,93 (Diop, 2009). Par contre, l'huile provenant des fruits noirs présente une densité plus élevée. On observe donc une légère augmentation de la densité avec la maturation des fruits. Selon le Comité des Constructeurs Français d'Automobiles (CCFA, 2006), la densité du gazole est de l'ordre de $0,85 \%$. Ainsi, d'une manière générale, l'huile de jatropha présente une densité légèrement supérieure à celle du gazole et ne répond donc pas aux spécifications qui permettent aux moteurs diesel de respecter les très bas niveaux d'émissions polluantes imposés par les normes (CCFA, 2006).

Ainsi, les caractéristiques physicochimiques des graines et de l'huile varient suivant le degré de maturité du fruit. Il ressort que, à part la densité de l'huile, les caractéristiques des graines et de l'huile sont meilleures pour les graines issues de fruits noirs et moins intéressantes pour les graines issues d'autres types de fruits.

\section{Conclusion}

L'étude des caractéristiques physicochimiques des graines de pourghère et de leurs huiles révèle que, excepté la densité de l'huile, les caractéristiques s'améliorent progressivement au cours de la maturation des fruits et que le stade de fruits noirs correspond aussi aux meilleures qualités de la graine et de l'huile. Ainsi, en dehors de tout processus de séchage adéquat, la récolte des fruits noirs serait la meilleure option.

\section{CONFLIT D'INTERET}

Nous déclarons qu'il n'y a aucun conflit d'intérêt entre les auteurs de ce manuscrit.

\section{CONTRIBUTIONS DES AUTEURS}

GNRH a été l'investigateur principal ; $\mathrm{CBG}$ et $\mathrm{APH}$ ont conçu le protocole 
expérimental et supervisé l'ensemble des travaux; MC-C a coordonné les travaux de terrain. $\mathrm{FG}$ et $\mathrm{AH}$ ont respectivement supervisé et réalisé les travaux de laboratoire.

\section{REFERENCES}

Abidin R, Sepidar S, Yunus ZZ, Azhari M. 2009. Extraction of Oil from Jatropha Seeds-Optimization and Kinetics. American Journal of Applied Sciences. 6(7): 1390-1395. DOI : 10.3844/ajassp. 2009.

Ahoton LE, Quenum F. 2012. Floral biology and hybridization potential of nine accessions of physic nut (Jatropha curcas L.) originating from three continents. Tropicultura, 30(4): 193-198.

Ahoton LE, Quenum F, Mergea G. 2011. Evaluation agro-morphologique et sélection des meilleures accessions de pourghère (Jatropha curcas L.) introduites au Bénin. Int. J. Biol. Chem. Sci., 5(4): 1619-1627.

Akowuah JO, Addo A, Kemausuor F. 2012. Influence of storage duration of Jatropha curcas seed on oil yield and free fatty acid content. ARPN Journal of Agriculture and Biological Science, 7(1): 41-45.

Assogbadjo AE, Amadji GR, Glèlè Kakai R, Mama A, Sinsin B, Van Damme P. 2009. Evaluation écologique et ethnobotanique de Jatropha curcas L. au Bénin. Int. J. Biol. Chem. Sci., 3(5), 1065-1077.

Carels N. 2009. Jatropha curcas: a review. Adv. Bot. Res., 50: 39-86. DOI: 10.1016/s0065-2296(08)00802-1

Chisti Y. 2007. Biodiesel from microalgae. Biotechnol. Adv., 25: 294-306. DOI:10.1016/ j.biotechadv.2007.02.001

Diop M. 2009. Production de biodiesel à partir de l'huile de Jatropha. Projet de fin d'étude pour l'obtention du Diplôme d'Ingénieur de conception en Electromécanique, Ecole Supérieure Polytechnique, Centre de Thiès,
Université Cheikh Anta Diop, Sénégal, $135 \mathrm{p}$.

FAO/ FIDA. 2010. Jatropha : une culture bioénergétique pour les petits agriculteurs. Rapport du 22 juillet 2010, Rome. http://www.fao.org, (Mars 2012).

Ferrao JEM, Ferrao AMBC, Patricio MTS. 1982. Purgueira da Ilha do Fogo. Composicao da sementa, algumas caracteristicas da gordura [Z]. Universida de Tecnica de Lisboa, Instituto Superior de Agronomia, Seccao de Agronomia Tropical. Estudos, No. 14.

Gandonou ChB, Houmba NR, Desquilbet S, Fakambi K, Datinon B, Marshall E. 2012a. Evaluation de la levée et de la croissance chez douze accessions de pourghère (Jatropha curcas) au Bénin, Bulletin de la Recherche Agronomique du Bénin, 12: 12-18.

Gandonou ChB, Houmba NR, Desquilbet S, Fakambi K, Datinon B, Marshall E. 2012b Caractérisation agromorphologique de plantations de pourghère (Jatropha curcas L.) soumises à différents itinéraires techniques au centre du Bénin, Revue Spéciale Journées Scientifiques de la Faculté des Lettres, Arts et Sciences Humaines (FLASH/UAC), 1(3).

Gui MM, Lee KT, Bhatia S. 2008. Feasibility of edible oil vs. non-edible oil vs. waste edible oil as biodiesel feedstock. Energy, 33: 1646-1653. DOI:10.1016/j.energy. 2008.06.002.

Henning RK. 2007. Jatropha curcas L. In Medicinal Plants/Plantes Médicinales 1, van der Vossen HAM, Mkamilo GS (eds). Prota 11(1). [CD-Rom]: Wageningen, Pays-Bas. http://database. prota.org/PROTAhtml/Jatropha\%20curca s_Fr.htm, (02/10/2010).

Jongschaap REE, Corre WJ, Bindraban PS. 2007. Claims and Facts on Jatropha curcas. Global Jatropha curcas Evaluation, Breeding and Propagation 
Program. Plant Research International: Wageningen; 35p.

Kabutey A, Ceska Z, Katedra M. 2010. Screw press performance for oil extraction from Jatropha curcas L. seeds of different moisture content. Scientia Agriculturae Bohemica, 41(4): 225-230.

Minengu J de D, Mobambo P, Mergeai G. 2014. Influence de l'environnement et des pratiques culturales sur la productivité de Jatropha curcas L. en Afrique subsaharienne (synthèse bibliographique). Biotechnologie, Agronomie, Société et Environnement, 18(2): 290-300.

Rehm S, Espig G. 1991. The Cultivated Plants of the Tropics and Subtropics. Verlag Josef Margraf, Weikersheim; 185-186.

Sarin R, Sharma M, Sinharay S, Malhotra RK. 2007. Jatropha-palm biodiesel blend: An optimum mix for Asia. Fuel, 86: 13651371. DOI:10.1016/j.fuel.2006.11.040

Sirisomboon P, Kitchaiva P. 2008. Physical properties of Jatropha curcas L. kernels after heat treatment. Biosystems Engineering, 102(2): 244-250. DOI: 10.1016/j.biosystemseng.2008.11.003
Stavarache C, Vintoru M, Maeda Y, Bandow H. 2007. Ultrasonically driven continuous process for vegetable oil transesterification. Ultrasonic Sonochem 14: 413-417.

Tiwari AK, Kumar A, Raheman H. 2007. Biodiesel production from jatropha oil (Jatropha curcas) with high free fatty acids: An optimized process. Biomass and Bioenergy, 31: 569-575. DOI: 10.1016/j.biombioe.2007.03.003.

Veljkovic VB, Lakicevic SH, Stamenkovic OS, Todorovic ZB, Lazic KL 2006. Biodiesel production from tobacco (Nicotiana tabacum L.) seed oil with a high content of free fatty acids. Fuel, 85: 2671-2675. DOI:10.1016/j.fuel. 2006.04.015.

Val Biom, 2005. http://www.notreplanete.info/actualites/actu_827_huile_ve getale_voiture.php (consulté le 14 décembre 2015). Prénorme DIN 51605 